\title{
Sistema de Recomendação Educacional para Mobile: um foco na Experiência do usuário
}

\author{
Cristina Alba Wildt Torrezzan /UFRGS - crisawt@ gmail.com \\ Adalto Selau Sparremberger / FADERGS |SENACRS - tec.adalto@gmail.com \\ Ketia Kellen Araújo da Silva / UFRGS - ketiakellen@ gmail.com \\ Patricia Alejandra Alejandra Behar / UFRGS - pbehar@ terra.com.br \\ José Américo Lacerda Félix / UFRGS - joseamericolf@gmail.com \\ Marcela Futuro de Carli / UFRGS - marcelafuturoc@ gmail.com
}

\begin{abstract}
Resumo: O presente artigo aborda o desenvolvimento de um sistema de recomendação Educacional (SRE) para mobile denominado RecOAComp. A partir da utilização da versão web RecOAComp em disciplinas de pós-graduação, verificou-se o perfil digital de docentes e discentes e sua predileção pelo uso de tecnologias móveis. Dessa forma, visualizou-se a necessidade de implementar uma nova versão do recomendador no formato de um aplicativo contemplando especificidades relacionadas à experiência do usuário, como a usabilidade e acessibilidade. A partir do planejamento do caráter comunicativo e técnico das interfaces e da metodologia utilizada na elaboração do aplicativo, baseada no Design Thinking, foi construída a versão mobile, priorizando o design educacional a partir dos fatores técnicos, gráficos e pedagógico.
\end{abstract}

Palavras-Chave: Sistema de Recomendação, Aplicativo Educacional, Design de Interface Mobile.

Educational Recommendation System for Mobile: A Focus on User Experience

\begin{abstract}
This paper deals with the development of an Educational recommendation system (ERS) for mobile called RecOAComp. From the use of the web version of RecOAComp in postgraduate disciplines, we verified the digital profile of teachers and students and their preference for the use of mobile technologies. Thus, we saw the need to implement a new version of the referred adviser in the form of an application addressing specificities related to usability and accessibility. From the planning of the communicative and technical character of the interfaces and the methodology used in the elaboration of the application, based on Design Thinking, the mobile version was built, prioritizing the educational design based on the technical, graphic and pedagogical factors.
\end{abstract}

Keywords: Educational App, Recommendation System, Mobile Interface Design.

\section{Introdução}

$\mathrm{O}$ artigo tem com objetivo apresentar o processo de desenvolvimento de um sistema de recomendação para mobile denominado RecOAComp. Foi verificada a necessidade da sua elaboração a partir da aplicação da versão web do referido recomendador em duas turmas de pós-graduação de uma universidade pública. A maioria dos alunos o acessaram através de dispositivos móveis, embora houvesse computadores à disposição na classe. Problemas relacionados à usabilidade e acessibilidade foram encontrados, sinalizando a necessidade de convertê-lo em um aplicativo mobile. Nesse sentido é que foi motivado o presente estudo. 
Portanto, com a crescente utilização de dispositivos móveis em tarefas cotidianas na sociedade atual, verifica-se o surgimento de uma tendência relacionada à transformação de sistemas web em versões para smartphones e tablets. No entanto, por vezes, as adaptações realizadas para navegadores móveis, também conhecidos como sites responsivos, não são suficientes para proporcionar uma boa experiência ao usuário. Esse fato ocorre, principalmente, devido à diminuição do tamanho da tela e à nova lógica e formato navegacionais, baseados no toque e não mais no uso do mouse.

Do ponto de vista pedagógico, essa relação demonstra-se importante para apoiar o processo de construção do conhecimento do sujeito. A exploração das interfaces e a investigação do conteúdo educacional precisam ocorrer de forma intuitiva e lógica, fornecendo confiança ao aluno. Inconsistências nessa interação podem gerar uma carga cognitiva extra ao discente, referente ao uso do recurso digital, além da já envolvida no processo de ensino e aprendizagem dos conteúdos educacionais por ele abordados. Segundo Santos e Leite (2019), os estudantes estão sempre conectados à internet e fazem tudo de maneira rápida, além de conviverem com a tecnologia diariamente realizando diversas tarefas ao mesmo tempo. Assim, entende-se a importância de construir recursos/ aplicativos que auxiliem tanto aluno quanto docente no processo de ensino e aprendizagem.

Nesse sentido, pode ser observado que a construção de aplicativos está superando a elaboração de sites responsivos. Um dos principais motivos é o planejamento da experiência do usuário segundo as necessidades de uma tecnologia específica (dispositivo móvel), ao contrário de abrandá-la entre aparelhos distintos (computador e dispositivo móvel). No caso particular de aplicativos, novos princípios de UX precisam ser abordados. A Experiência do Usuário (UX - User Experience) refere-se às percepções e respostas de uma pessoa, a partir da utilização (ou antecipação do uso) de um produto ( ISO 9241-210). Dentre os princípios definidos por Griffiths (2015), pode-se citar: (1) eliminar obstáculos de utilização; (2) simplificar decisões de conversão; (3) proporcionar o máximo de conveniência; (4) self-service, engajamento e satisfação (ou encantamento) e (5) higiene de usabilidade (evitar que os usuários pensem sobre coisas que deveriam ser simples). Nesse sentido, embora a construção de aplicativos possa envolver um nível maior de complexidade em relação à elaboração de outros tipos de recursos digitais (XANTHOPOULOS e XINOGALOS, 2013), trata-se de uma importante tecnologia de apoio ao perfil do sujeito Mobile (KRIMBERG, 2019), presente na sala de aula.

Seguindo essa perspectiva, este artigo apresenta os procedimentos metodológicos realizados para a idealização e construção do aplicativo proposto. Nesse sentido, encontra-se organizado em 5 seções, sendo esta primeira a introdução. As seções 2 e 3 , abordam o referencial teórico, no caso o sistema de recomendação RecOAComp e o Planejamento do Design Educacional para aplicativos e suas funcionalidades. Na seção 4, é apresentada a metodologia, através dos procedimentos utilizados para a construção do aplicativo. Os resultados são elencados na Seção 5 e por fim, na seção 6 são abordadas as considerações finais.

\section{Sistema de Recomendação RecOAComp: Funcionamento e Aplicações}

O sistema de recomendação educacional RecOAComp indica objetos de aprendizagem (OAs) a partir das competências que o usuário necessita desenvolver e/ou aprimorar. O professor cadastra a sua atividade de ensino, vinculando à ela as competências que serão abordadas. Também as associa a objetos de aprendizagem, informando o quanto cada um deles apóia o desenvolvimento das competências elencadas. O aluno, ao acessar o sistema 
pela primeira vez, preenche um formulário de autoavaliação, informando o quanto acredita ter de cada competência indicada. Esse será o seu perfil de competências, que poderá ser atualizado em diferentes momentos durante a atividade pelo aluno e servirá como base para as recomendações realizadas pelo sistema. Quando solicitar recomendação, o sistema cruza o seu perfil de competências com a dos objetos de aprendizagem vinculados à disciplina, indicando os mais adequados às suas necessidades educacionais. O objetivo é apoiar o processo de ensino e aprendizagem do sujeito, evitando o tempo e esforço normalmente despendidos em buscas na internet por materiais relevantes aos seus estudos.

Em 2018, realizou-se uma remodelagem do design gráfico e de algumas partes do fluxo navegacional. Como forma de avaliar esses aprimoramentos, a versão web do RecOAComp foi utilizado em duas disciplinas de pós-graduação em educação e informática na educação, nos semestres 2018/2 e 2019/1, totalizando 50 alunos. Em um primeiro momento, estes utilizaram o sistema na função de discente, apenas solicitando recomendações para a realização de atividades propostas. Logo depois, ao final das disciplinas, atuaram no papel de professores, cadastrando atividades de ensino, objetos de aprendizagem, competências e definindo os vínculos entre eles. Por fim foi aplicado um questionário aos alunos, instigando sobre a experiência de uso do RecOAComp web. Como resultado, todos consideraram relevante o uso do referido recomendador na sala de aula, tanto do ponto de vista docente, quanto discente. Julgaram as interfaces como objetivas, cleans, de modo a comunicar claramente as informações. Quanto à lógica de navegação, tiveram um pouco de dificuldade em definir qual procedimento deveria realizar primeiro: cadastrar a atividade de ensino, as competências ou os objetos de aprendizagem. Também sugeriram que, no formulário de autoavaliação, as competências analisadas ficassem visualmente marcadas, auxiliando o usuário a identificar as que ainda precisavam ser verificadas. Esses elementos foram aprimorados no sistema.

Porém, durante a aplicação do RecOAComp nas duas turmas, um fato foi destacado. Cerca de $60 \%$ dos alunos preferiram acessá-lo através do seu smartphone ou tablet, mesmo havendo computadores disponíveis para todos na sala de aula. Nesse sentido, ocorreram incompatibilidades referentes à experiência do usuário, como: dificuldade de visualizar todas as informações de cada tela (necessidade de rolagem constante), fonte pequena, ícones difíceis de serem clicados com sucesso, alguns campos dos formulários de cadastro não eram visualizados ou preenchidos corretamente.

Logo, através dessa prática, verificou-se a preferência dos alunos, nas duas turmas, pela utilização de dispositivos móveis pois, diariamente, também acompanhavam o conteúdo e as discussões das aulas através de seus celulares (site da disciplina). À vista disso, optou-se por elaborar uma versão Mobile do RecOAComp, como forma de atender essa preferência emergente na educação e possibilitar uma boa experiência ao usuário. Entretanto, a construção e planejamento do design educacional para aplicativos é uma etapa importante desse processo, portanto é tratada na seção a seguir.

\section{Planejamento do Design Educacional para aplicativos}

O termo design pode ser entendido como o ato de projetar, inovar, desenvolver (SILVESTRI, 2018). Com relação às interfaces gráficas, envolve não apenas concepções gráficas, como também objetiva contemplar fatores relacionados à interação homemmáquina, como os de usabilidade e acessibilidade. No âmbito educacional, essas preocupações são ainda maiores, pois as interfaces servem de meio para o acesso e discussões de conteúdos científicos complexos. Dessa forma, a sua utilização e entendimento não podem exigir, do usuário, uma carga cognitiva extra. Nesse sentido, o 
design educacional trata de fatores técnicos, gráficos e pedagógicos durante a elaboração de interfaces pedagógicas. Uma das principais intenções é utilizar um design visual que se relacione com o perfil do usuário (afetividade e identidade) e auxilie na compreensão dos conteúdos estudados. Já no contexto da aprendizagem móvel, inclui novos elementos, relacionados com o tamanho reduzido da tela de visualização e as habilidades motoras necessárias para a sua exploração.

A aprendizagem móvel, originada do termo mobile learning, ou ainda, MLearning, significa a combinação entre as tecnologias de comunicação e informação (TIC) com as móveis, por exemplo, smartphones e tablets, possibilitando a construção do conhecimento em distintos momentos e locais (UNESCO 2014). Essa prática demonstrase importante, devido à disseminação cada vez maior do uso desses equipamentos móveis nas ações cotidianas dos indivíduos. Na educação, portanto, verifica-se, a relevância em compreender o perfil do sujeito mobile (KRIMBERG, 2019), que se refere aos sujeitos que estão acostumados a utilizar os aplicativos móveis como meio para realizar suas tarefas cotidianas. Um dos principais atrativos dessas plataformas tecnológicas é a rapidez com que a informação é acessada e as necessidades dos indivíduos são atendidas. Assim, tem-se como características desse perfil, a velocidade, conectividade, imersão nas mídias sociais e atuação multitarefa (KRIMBERG, 2019). Seguindo esse contexto, verifica-se uma quantidade cada vez maior de sujeitos mobiles na sala de aula. No entanto, por vezes, as práticas pedagógicas ainda são predominantemente baseadas em metodologias tradicionais, fundamentadas na ação pacífica dos alunos. Por outro lado, a simples utilização de dispositivos móveis na educação não é garantia de uma trajetória de aprendizagem crítica, construtivista e motivadora. Portanto, entende-se que é necessário aplicar estratégias pedagógicas que instiguem os discentes à busca do conhecimento e os auxilie a saber interagir com essa tecnologia móvel de forma responsável e produtiva. Nesse sentido, o planejamento das interfaces educacionais de aplicativos voltados ao mlearning pode ser focado na experiência do usuário, no intuito de apoiar esses objetivos educacionais. Dessa forma, a elaboração do recomendador para mobile RecOAComp baseou-se nos parâmetros para a elaboração de aplicativos educacionais com foco no sujeito mobile, desenvolvido por Krimberg (2019), elencados a seguir:

\section{Parâmetros Pedagógicos}

- Apresentar inicialmente o conteúdo mais relevante

- Permitir o acesso ilimitado aos conteúdos do app

- Dividir os conteúdos em pequenos módulos

- Apresentar os conteúdos do modo linear

- Utilizar textos reduzidos, vídeos de curta direção, animações, infográficos, podcasts e áudios explicativos

- Definir os objetivos de aprendizagem e o público-alvo,

- Desenvolver atividades e conteúdos interligados aos interesses pessoais dos estudantes

- Disponibilizar o conteúdo em linguagem acessível

- Uso de atividades e conteúdos vinculados a seus interesses pessoais

- Definir o tipo de comunicação (síncrona/assíncrona)

- Planejar um espaço para o compartilhamento de informações (dúvidas, ideias) entre alunos e professores

Parâmetros Técnicos

- Disponibilizar um endereço de download de fácil acesso

- Compatibilidade com diversos sistemas operacionais 
- Definir os recursos de hardware que serão utilizados

- Possibilitar uso offline do aplicativo

Parâmetros de Usabilidade

- Utilização harmônica de cores e fontes, segundo a identidade visual do aplicativo

- Análise da utilização de menus, botões, links, rolagem de tela e entrada de texto

- Organização das informações de maneira clara

- Permissão da customização da interface: tamanho de letras e uso de cores

- Planejamento dos comandos gestuais a serem utilizados

- Adaptação dos conteúdos ao tamanho de tela

- Utilização de metáforas conhecidas pelo público-alvo

- Planejamento do manuseio descomplicado das funções do aplicativo

Com base nestes critérios, foram organizadas e executadas as etapas que compõem a metodologia do presente estudo.

\section{Metodologia}

A versão Mobile do recomendador RecOAComp baseou-se nos parâmetros para a elaboração de aplicativos educacionais com foco no sujeito mobile, desenvolvido por Krimberg (2019). A metodologia utilizada foi o Design Thinking que, de acordo com a IDEO (2018) e Fernandes (2016), é o modelo mais adequado para aplicação de projetos educacionais. Assim, foi utilizado no desenvolvimento do aplicativo o modelo de cinco fases: 1. Descoberta: ocorre a identificação do problema a ser solucionado, dos seus elementos e a criação de hipóteses sobre como abordá-lo.; 2. Interpretação: realiza-se uma interpretação sobre o aprendizado realizado na etapa 1; 3. Ideação: analisa-se possibilidades de criação a partir de oportunidades visualizadas; 4. Experimentação: caracteriza-se pela concretização da ideia (prototipação) e 5. Evolução: ocorre a experimentação do protótipo e a análise de possíveis ajustes e aprimoramentos. Também, para o acompanhamento do processo de desenvolvimento, foi incorporado um quadro Kanbam1, no software Trello2, contendo a relação de funcionalidades a serem desenvolvidas em cada sprints3. A construção foi realizada através de uma equipe interdisciplinar, constituída por pedagogos, designers e programadores. Durante o processo foram realizadas reuniões quinzenais com o grande grupo, onde eram compartilhadas as sugestões, desafios e decisões específicas de cada área, para planejamentos pontuais e, posteriormente, debatidos nas reuniões gerais para aprovação. Também foram utilizados princípios do design de experiência do usuário e acessibilidade (GRIFFITHS, 2015). O intuito foi possibilitar que as interfaces elaboradas apoiassem as ações e necessidades educacionais do usuário, segundo o perfil do sujeito mobile. Na seção a seguir é apresentada a aplicação da metodologia nos procedimentos de elaboração das interfaces e implementação técnica do aplicativo.

\footnotetext{
${ }^{1} \mathrm{O}$ quadro Kanban permite um controle detalhado de produção com informações sobre quando, quanto e o que produzir.

2 Trello é uma ferramenta de gerenciamento de projetos colaborativo online e pode ser acessada através do link: https://trello.com

${ }^{3}$ Sprint é um conjunto de tarefas que devem ser executadas/desenvolvidas em um período pré-definido de tempo.
} 


\section{Concepção das Interfaces Móveis e Implementação Técnica do Aplicativo}

Com base na metodologia Design Thinking, voltada à resolução de problemas e à inovação, foram realizadas 5 etapas, conforme detalhadas a seguir.

Etapa 1 - Descoberta: Nesta, verificou-se a necessidade de usar o recomendador RecOAComp em dispositivos móveis, a partir das especificidades do perfil do sujeito mobile. A versão web não era compatível em alguns aspectos, principalmente naqueles referentes à experiência do usuário. O desenvolvimento das telas do aplicativo foi baseado em princípios relativos ao design de experiência e de interface para o usuário, conforme apresentado acima, a partir de 5 etapas. Na primeira, de descoberta, foi realizada a definição do objetivo, que foi transformar um sistema de recomendação versão web para mobile.

Etapa 2 - Interpretação: Dessa forma, definiu-se o perfil do usuário (sujeito mobile) do aplicativo do recomendador e os fatores que precisariam ser contemplados, novamente referentes à UX. Assim, foram considerados os fluxos de navegação, as ferramentas necessárias para a execução de cada função presente, a acessibilidade - em especial para pessoas com baixa visão - , e uma boa organização das informações dispostas, tornando, desta forma, a utilização do aplicativo agradável e convidativa ao usuário. O desenvolvimento do aplicativo passa por diversas etapas e aperfeiçoamentos até sua conclusão.

Etapa 3 - Ideação: Tendo como base as funcionalidades e ações disponibilizadas nas interfaces da versão web do recomendador, idealizou-se, através de croquis, o design das telas do aplicativo. Utilizou-se como referência, princípios de experiência do usuário, usabilidade e acessibilidade, além dos parâmetros para a construção de aplicativos educacionais para o sujeito mobile. Também se concebeu o mapa de navegação do app e a escolha do framework Ionic ${ }^{4}$ para a realização da implementação técnica. Em seguida foram realizadas análises das telas versão web do sistema de recomendação, a fim de que haja um entendimento de quais funções estão presentes em cada tela, para que assim, elas sejam transpostas para o aplicativo e traduzidas para a linguagem mobile - a partir de pesquisas e da busca por referências em outros aplicativos que possuem suas usabilidades otimizadas.

Etapa 4 - Experimentação: O processo, portanto, avança para o desenho de rafes (esboços), feitos a mão com papel e lápis com as propostas para cada tela, conforme Figuras 1 e 2, apontando as funções presentes em cada uma delas, e a localização de cada botão interativo, respeitando os padrões e as ferramentas disponibilizadas pela plataforma Ionic, usada pela equipe de programação no desenvolvimento do aplicativo.

\footnotetext{
${ }^{4}$ É um framework para desenvolvimento de aplicações mobile híbridas, criado em 2013 pela Drifty e com um grande suporte da comunidade.
} 

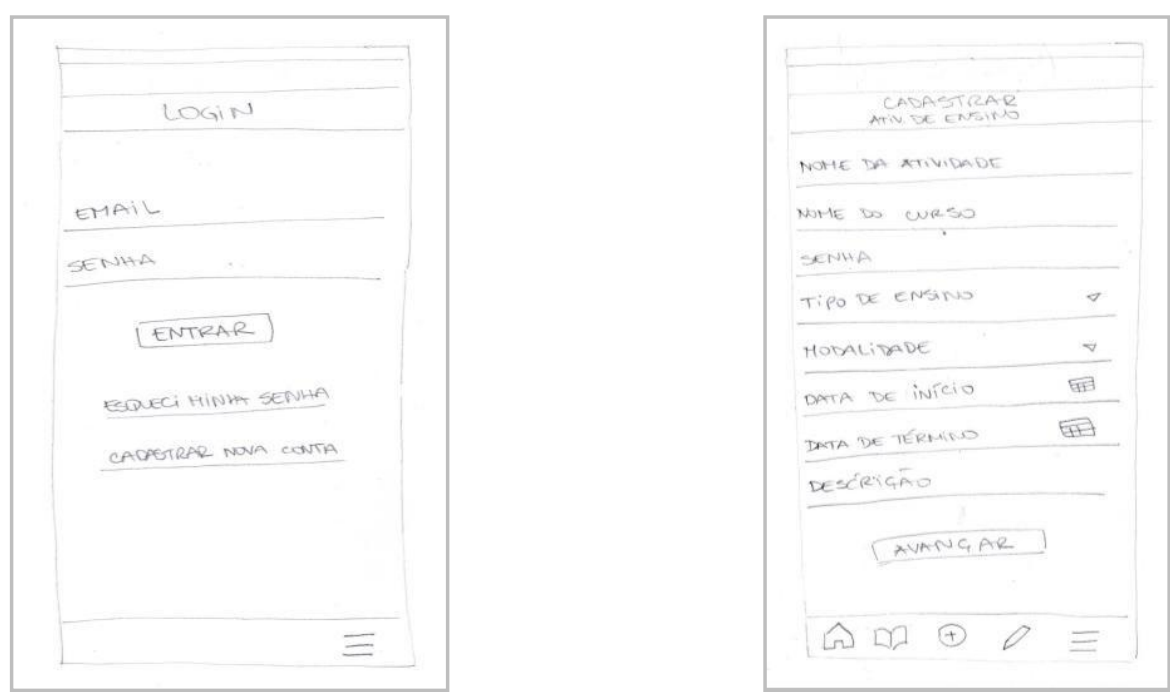

Figuras 1 e 2 - Rafe Tela Login e Rafe Tela Cadastro de Atividade de Ensino

Finalizados e aprovados os rafes, as telas foram construídas digitalmente nos softwares Adobe Illustrator e Adobe XD, com o auxílio do pacote de componentes do padrão Ionic para o design de interfaces desenvolvido e disponibilizado pela designer Barbara Wolfe (2019), para assim, aproximar-se do visual final e prototipar o aplicativo. O programa Adobe XD ainda permite a inserção de vínculos entre as telas, possibilitando a execução da sequência de navegação entre elas. As telas podem ser vistas nas Figuras 3 e 4.
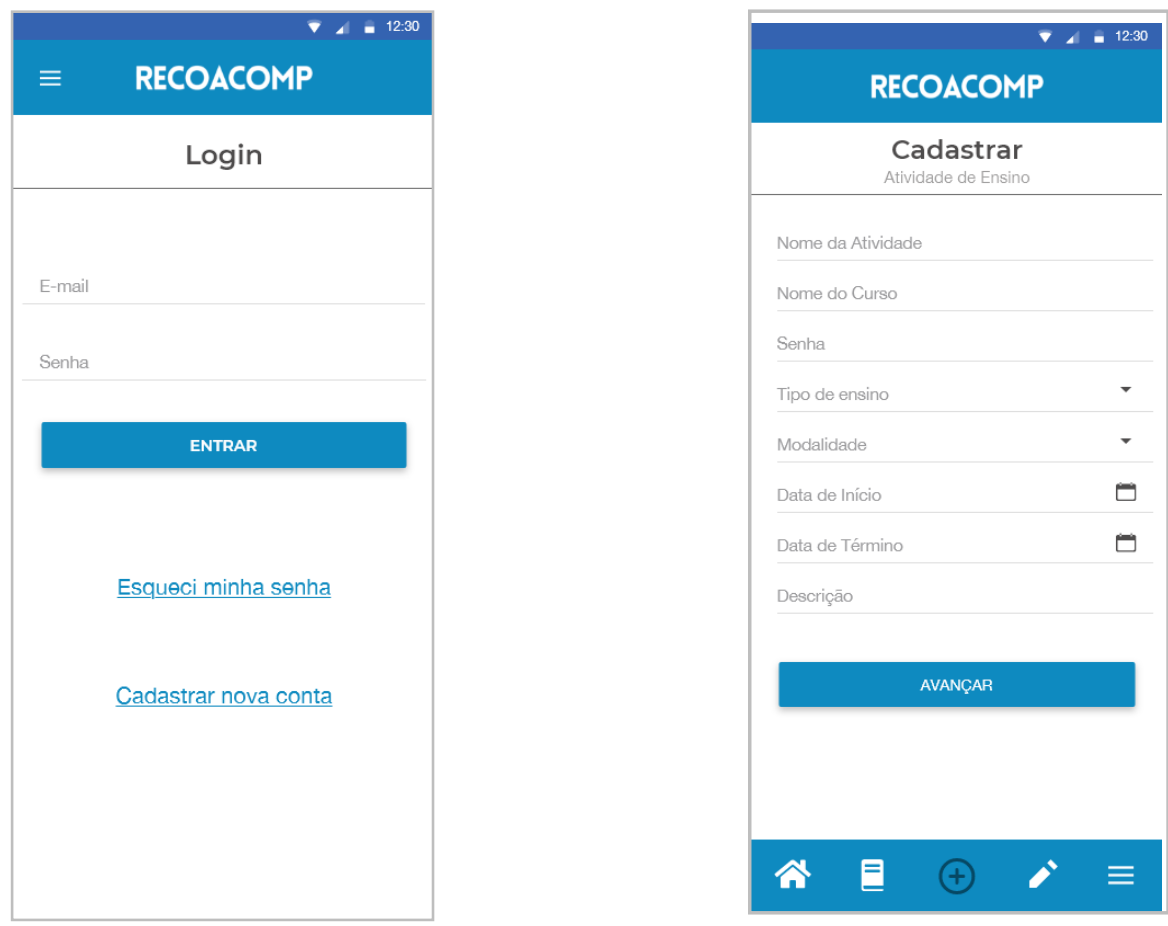

Figuras 3 e 4 - Protótipo Tela Login e Protótipo Tela Cadastro de Atividade de Ensino

Para a paleta cromática, foi optado por manter o padrão da versão web, com azul - cor geralmente aplicada em espaços de ensino (virtuais ou não) pois sugere tranquilidade, seriedade e conhecimento - , branco, preto e cinzas escuros, para que não houvesse prejuízos à acessibilidade. A família tipográfica escolhida foi a Helvetica Neue, 
por sua boa legibilidade e adequação em aplicativos mobile.

Também na Etapa 4, foi realizada a implementação do aplicativo para os dois sistemas operacionais, iOS e Android, decidindo-se, assim, que seria utilizada uma plataforma de desenvolvimento híbrido ${ }^{5}$. Após uma análise relacionando as ferramentas disponíveis no mercado, foi escolhido o framework Ionic. O framework Ionic mostrouse mais adequado a proposta do aplicativo RecOAComp pela facilidade de seu uso e rapidez no seu fluxo de desenvolvimento, oriundos do fato de ser um framework para criação de aplicativos híbridos. Sendo despendido o esforço do desenvolvimento apenas uma vez e o aplicativo disponibilizado para os sistemas operacionais Android e iOS. Entende-se também que existe uma pequena perda de performance ocasionada pelo uso de um framework de desenvolvimento híbrido de aplicativos, mas que pela arquitetura do projeto, pode ser desconsiderada.

O SRE, em sua versão web, já possui uma base de dados, com usuários, competências, atividades e objetos de aprendizagem cadastrados. Para o desenvolvimento do protótipo, não foi necessário realizar nenhuma alteração nesta base de dados. Sendo assim, foi preciso apenas o desenvolvimento de uma $\mathrm{API}^{6}$, utilizando o interpretador Javascript NodeJs ${ }^{7}$, para permitir a comunicação do protótipo do aplicativo com a base de dados já existente. Esta centralização dos dados, com o desenvolvimento da API, possibilitou a utilização da mesma lógica empregada na versão web no processo de recomendação dos objetos de aprendizagem.

No início da utilização do framework Ionic, a equipe de desenvolvimento teve algumas dificuldades pois ele engloba um conjunto de tecnologias: HTML, CSS, JavaScript, Angular e Cordova. Inicialmente, como forma de maximizar o conhecimento da equipe de desenvolvimento, foi construído um pequeno aplicativo de demonstração, para testes da plataforma de desenvolvimento. Com isto, verificou-se que o framework Ionic possui boa documentação de referência, que ajuda de forma expressiva no processo de implementação de aplicativos. Os aplicativos desenvolvidos no framework Ionic apresentam sempre estruturas de pasta semelhante, o que facilita o desenvolvimento de aplicativos na plataforma. O Ionic é dividido em módulos, permitindo a instalação de cada módulo conforme a necessidade de cada projeto. Porém, dependendo com computador utilizado para o desenvolvimento, este processo de instalação dos módulos pode ser um pouco demorado. Para que os projetos de aplicativos se tornem possíveis de serem executados em smartphones, também deve-se instalar módulos para isto.

Para prover a conexão do aplicativo com a API no servidor web do SRE, utilizouse uma aplicação construída em JavaScript com NodeJS. O protótipo foi implementado seguindo o projeto de Itextit\{layouts\} das telas, desenvolvido pela equipe de designers do projeto apresentado neste trabalho. Uma das vantagens em utilizar o desenvolvimento híbrido, em especial o Ionic, é que a aparência das telas, nos diferentes sistemas operacionais em que o aplicativo for executado, é demasiadamente semelhante. Isto pode ser verificado na Figura 5, que apresenta a tela Atividade, sendo executada respectivamente no iOS e no Android.

\footnotetext{
${ }^{5}$ No desenvolvimento híbrido, utiliza-se uma ferramenta de desenvolvimento específica para criar um modelo único que seja compatível com ambas as plataformas.

${ }^{6}$ Termo em inglês que significa Application Programming Interface que é um conjunto de rotinas e padrões de programação para acesso a um aplicativo de software ou plataforma baseado na Web.

${ }^{7}$ É uma plataforma construída sobre o motor JavaScript do Google Chrome para facilmente construir aplicações de rede rápidas e escaláveis.
} 


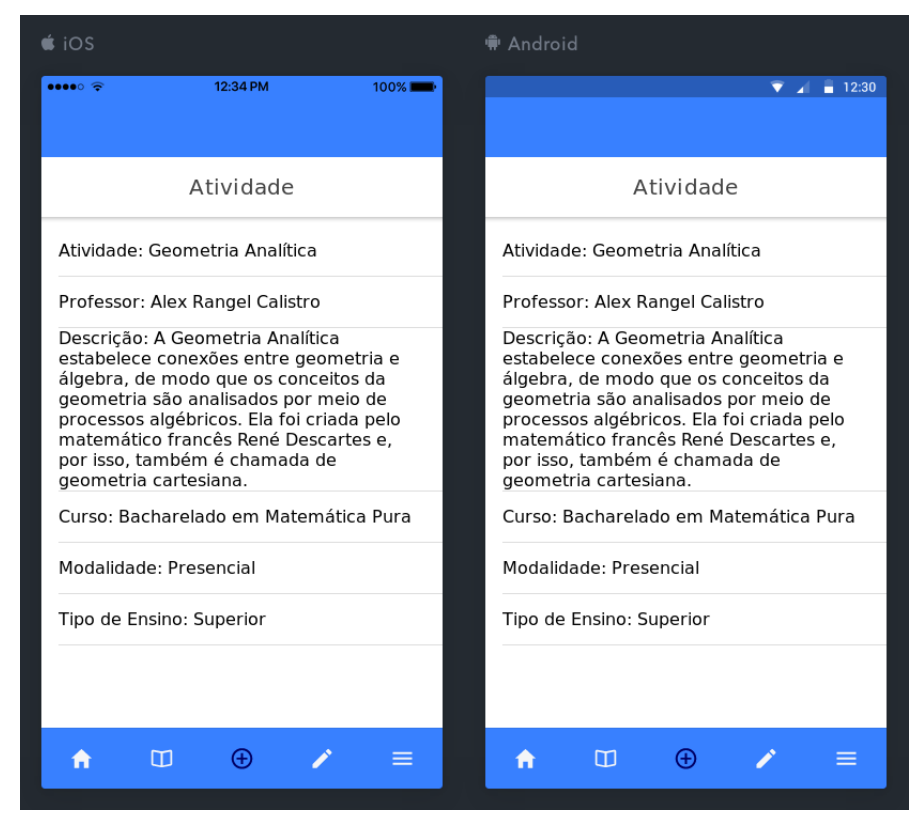

Figura 5 - Tela Atividade sendo executada nas plataformas iOS e Android

Etapa 5 - Evolução: A partir da testagem dos fluxos de navegação entre as telas, no programa Adobe XD, foram realizados aperfeiçoamentos nos elementos de algumas interfaces. Dentre elas a localização de ícones e informações, inserção de textos de ajuda, redução da quantidade de informação de determinadas interfaces dividindo em duas ou mais telas, entre outros. Os resultados passam por contínuas reavaliações e aprimoramentos até sua versão final.

\section{Considerações Finais}

Este artigo apresentou o processo de concepção das interfaces gráficas e implementação técnica de um aplicativo constituído por um sistema de recomendação educacional. A motivação ocorreu a partir da utilização da versão web do referido recomendador em duas disciplinas de pós-graduação. Nessas práticas, visualizou-se a preferência dos alunos em acessá-lo através de dispositivos móveis. No entanto, o sistema demonstrou inconsistências, principalmente relacionadas à experiência do usuário. Por esse motivo, optou-se pelo desenvolvimento do RecOAComp Mobile, de modo a buscar a contemplação de fatores gráficos e técnicos necessários para proporcionar uma utilização eficaz através da tecnologia móvel. Para a sua construção foi utilizada a metodologia Design Thinking, implementada através de uma equipe interdisciplinar, composta por pedagogos, designers e programadores. Primeiramente, a equipe pedagógica e técnica realizou rafes integrando os elementos e funcionalidades já existentes nas interfaces web com as necessidades das telas para dispositivos móveis. A identidade visual seguiu o padrão da versão web, integrando os componentes do padrão Ionic, para apoiar a implementação técnica. Ao mesmo tempo, a equipe técnica optou por utilizar o framework Ionic para a implementação do aplicativo. Após, as telas foram elaboradas através da utilização dos softwares Adobe Illustrator e Adobe XD, esse último possibilitando a análise do fluxo de navegação entre as interfaces. O mapa navegacional foi idealizado com base nas especificidades referentes à acessibilidade e usabilidade de aplicativos em dispositivos móveis. Com base nele e nas interfaces projetadas, iniciouse o processo de codificação do aplicativo, etapa em que se encontra atualmente. Em seguida, serão realizadas avaliações internas e externas. Após a sua validação, o aplicativo 
será publicado nas lojas de aplicativos PlayStore e AppStore. Dessa forma, pretende-se disponibilizar gratuitamente, à comunidade acadêmica em geral, o sistema RecOAComp em sua versão para dispositivos móveis, apoiando o perfil do sujeito mobile que está cada vez mais presente em atividades de ensino-aprendizagem.

\section{Referências}

FERNANDES, Kleber Tavares et al. Uma Experiência na Criação de Game Design de Jogos Digitais Educativos a partir do Design Thinking. RENOTE 16, 1 (2016), $1-10$

GRIFFITHS, Stephen. Mobile AppUX Principles: Improving user experience and optimising conversion.Google. 2015. Disponível em: $<$ https://d3atsf3fgek2rw.cloudfront.net/content/uploads/2015/04/Mobile-App-UXPrinciples_Full-Report_Final.pdf> Acessado em: 20 out 2019

IDEO. 2018. Design Thinking for Educators. Disponível em: <https://designthinkingforeducators.com/> Acessado em: 29 out 2019

ISO 9241-210. Ergonomics of human-system interaction - Part 210: Human-centred design for interactive systems. Disponível em: <https://www.iso.org/standard/52075.html > Acessado em: 29 out. 2019.

KRIMBERG, Laura. AppEduMobile: Parâmetros para a construção de aplicativos educacionais com foco no Sujeito Mobile. Dissertação (Mestrado em Educação). Faculdade de Educação da UFRGS. Ano de Obtenção: 2019, 175 f.

SANTOS, Cicero Ernandes de Melo; LEITE, Bruno Silva. Construção de um jogo educativo em uma plataforma de desenvolvimento de jogos e aplicativos de baixo grau de complexidade: o caso do Quizmica - Radioatividade. RENOTE, v. 17, n. 1, 2019.

SILVESTRI, Gabriel. O que é Design: quem são os Designers e quais são suas áreas de atuação. 2018. Disponível em: https://gabrielsilvestri.com.br/o-que-e-design/\#o-que-edesign. Acesso em 29 out. 2019.

UNESCO. Reading in the mobile era: a study of mobile reading in developing countries. (2014) Disponível em: <http://unesdoc.unesco.org/images/0022/002274/227436e.pdf>. Acesso em: 18 out. 2019.

XANTHOPOULOS, S.; XINOGALOS, S. A comparative analysis of cross-platform development approaches for mobile applications. In: ACM. Proceedings of the 6th Balkan Conference in Informatics. [S.1.], 2013. p. 213-220.

WOLFE, Barbara. UX projects. Disponível em: 〈https://www.barbara-wolfe.com/ionic $>$ Acesso em: 15 out. 2019. 